\title{
On the Timeliness of Multi-Hop Non-Beaconed ZigBee Broadcast Communications
}

\author{
Paulo Bartolomeu \\ DETI / DI \\ University of Aveiro / Micro I/O \\ 3810-193 Aveiro \\ bartolomeu@microio.pt
}

\author{
José Fonseca \\ DETI \\ University of Aveiro \\ 3810-193 Aveiro \\ jaf@ua.pt
}

\author{
Francisco Vasques \\ DEMEGI \\ University of Porto \\ 4200-465 Porto \\ vasques@fe.up.pt
}

\begin{abstract}
This paper studies the adoption of the ZigBee technology to replace broadcast-based wired communications in automation systems for Health Smart Homes. An analytical study is presented as well as the experimental results validating the derived expressions. Results show that the timeliness of ZigBee broadcast communications, in interference-free environments, can cope with the requirements of home automation applications and those found in building automation.
\end{abstract}

\section{Introduction}

The improvement of the quality of life of elderly and impaired people has been, for some time, in the agenda of governments as result of population aging and increased life expectancy, fuelled by improvements in medical care services and in technology [9]. The pressure for cost containment in health organizations and social care services is pushing the development of solutions to maintain elderly or impaired people living in their homes [6] while being assisted by intelligent systems.

The B-Live ${ }^{1}$ system [5] is a flexible and modular home automation solution designed to enable conventional homes to become Health Smart Homes, including the possibility to accommodate real-time and safety critical applications. The B-Live system includes control modules and interface devices.

Control modules are installed in appliances (doors, window blinds, etc.) to allow conventional and augmented operation. Interface devices are designed targeting elderly and impaired users. Modules communicate using the CAN fieldbus [8] in a producer-consumer scheme. The option for this technology is supported on its distinctive properties and add-ons related with fault tolerant operation, real-time communications and cost. Nevertheless, the use of a wired fieldbus poses several problems to the B-Live system. The first one regards the cost (both in time

\footnotetext{
${ }^{1}$ The B-Live system was awarded in 2007 with the Prize Eng. Jaime Filipe, a distinction given by the Portuguese Social Care Institute to the best innovative design promoting autonomy.
}

and labour) of installing wires. The second is related lack of flexibility of the wired connections. For example, if a user wishes to move a heater from a room to another, he/she must have new wires installed. The last aspect regards the aesthetics of having installed plastic conduits in the walls of the home. Concomitantly, the B-Live system would benefit considerably of using a wireless technology to support data communications.

\subsection{Requirements}

A common dwelling occupies an area of up to $150 \mathrm{~m}^{2}$, which requires either single hop communications, with ranges of some tenths of meters, or multi-hop communications, with a range of a few meters. Since the B-Live system targets the retrofitting of common homes, communication modules are installed in places where mains power is already installed (mains plugs, light switches, etc.) and, as such, energy requirements are not demanding. Nevertheless, if some devices become powered by batteries, energy consumption is an issue. Because the transmitted power is influenced by an exponential attenuation that increases with the distance between transmitter and receiver, short range communications offer a more efficient solution for transmitting data.

The topologies supported by the communication technology should ensure connectivity while providing an easy installation, low maintenance and good end-to-end performance. A common dwelling is usually organized in a per room basis and each room has a moderate number of appliances $(\leq 10)$ (window blind, door, mains plug, lamps, controls, etc.). As such, the communication technology must be able to route packets among different rooms and ensure that, if a routing node (installed in an appliance) becomes inoperable, a new route is automatically established and communications are maintained within the required bounds of latency and jitter. In the following text two B-Live usage scenarios are described.

\section{Daily Living Routine}

"A user comes home in a wheelchair and draws his/her mobile phone to open the front door using a menu-driven application. After having opened the door, still using the mobile phone application, the user enters the house, in- 
structs the door to close, turns ON the lights of the living room and orders the electrical window blinds to open. After, using the remote control, he/she turns on the TV set and watches his/her favourite program."

In this scenario, from a user perspective, the latency between issuing a command in the mobile phone and its effectiveness on the target appliance is important. The user may wait a second before seeing the window blind start to open but a similar delay for turning on the living room lamps will result in a bad user experience. As such, the wireless data communication shall be bounded by a $500 \mathrm{~ms}$ latency and a $100 \mathrm{~ms}$ jitter [22]. Moreover, since the envisaged event rate and amount of data per event are usually small, the required throughput is well bellow the limits of Wireless Personal Area and Wireless Local Area Networks (WPANs and WLANs, respectively).

\section{Alarm Scenario}

"An impaired user in a wheel chair detects the onset of a fire and gets in panic. He/she tries repeatedly to open the front door to escape the fire. At the same time, the B-Live fire detector generates an alarm, makes an emergency call to the fire-fighters and sends commands to open all doors in the home."

In this scenario, the occurrence of an alarm can trigger several events in a short period of time leading to the occurrence of high event rates (up to ten events per second) that must be handled within the defined timeliness bounds.

In this paper we evaluate the feasibility of using a ZigBee broadcast approach to replace the CAN fieldbus used in the B-Live system. Security and reliability concerns will not be addressed nor issues regarding power consumption. The (broadcast) event rate is assumed to be small, which is the most common scenario. High event rates are out of the scope of this paper.

This paper is organized as follows: section 2 introduces the ZigBee technology, presents a survey on the related work and justifies the use of the beaconless mode. Section 3 presents an analytical study of the broadcast latency bounds. The validation of the theoretical study is presented in section 4 where a test-bed is described and experimental results are discussed. This paper is concluded in section 5 by providing the summary of its findings as well as future work directions.

\section{Related Work}

ZigBee [2] is a communication technology designed for Low-Rate Wireless Personal Area Networks to be used in a wide range of applications such as home, industrial and environmental sensing and control. It was developed by the ZigBee Alliance [3] and specifies the Network and Application layers on top of IEEE 802.15.4 MAC and PHY $[14,12]$. The network layer allows multi-hop communication among Personal Area Network (PAN) joined devices by means of packet relaying. The formation of a network is managed by a coordinator assigning PAN addresses using an hierarchical tree structure scheme. The routing of packets among network devices is made using a technique similar to the Ad-hoc On-demand Distance Vector (AODV) routing protocol.

The IEEE 802.15.4 MAC layer defines two operation modes: beaconed and non-beaconed (beaconless). In beaconed mode, a superframe structure is used and slotted Carrier Sense Multiple Access with Collision Avoidance (CSMA/CA) is applied. In beaconless mode, a simple CSMA/CA mechanism is used instead. The IEEE 802.15.4 PHY layer defines 27 channels: 16 channels (at $250 \mathrm{kbps}$ each) in the Industrial Scientific and Medical (ISM) $2.4-2.4835 \mathrm{GHz}, 10$ channels (at $40 \mathrm{kbps}$ each) in the ISM $902-928 \mathrm{MHz}$ band, and one channel (at 20 kbps) in the $868.0868 .6 \mathrm{MHz}$ band. Two device types are supported: Full Function Device (FFD) and Reduced Function Device (RFD). A FFD is able to perform the role of coordinator (or router) and can communicate with any other devices in its radio range. An RFD is a simpler device that associates with a coordinator (or router) and is only able to communicate with FFD devices.

The propagation of broadcasts in Mobile Ad-Hoc Networks (MANETs) has been extensively studied in $[25,23$, 11] where work has been carried on to mitigate the issue identified by $\mathrm{Ni}$ et al. [20] as the broadcast storm problem. The main goals were to devise efficient, reliable, fast and simple broadcast algorithms resulting in a small redundancy, improved collision avoidance (from rebroadcasts) and autonomy [10]. However, the target application only shares a small subset of the MANET's characteristics.

The B-Live system addresses common dwellings and, as such, it is only required to cover a limited geographic area with a foreseeable reduced number of hops. Furthermore, since home appliances are (usually) static, the embedded wireless modules should not perform path discoveries often. Once the network system is deployed, devices become configured with a set of parameters that are almost static.

\subsection{Why using the ZigBee beaconless mode?}

The IEEE 802.15.4 performance has been studied in $[17,15,26]$. Both Lu et al and Zheng and Lee conclude that the packet delivery ratio of beaconless IEEE 802.15.4 is similar to that of IEEE 802.11. Zheng and Lee add that IEEE 802.15.4 shows advantages regarding control overhead and transaction latency. Furthermore, provided the higher power consumption and cost of IEEE 802.11 devices, an IEEE 802.15.4 based solution is a good option for low rate data applications. Lu et al also conclude that, for low duty cycle networks, such as the target one, a significant energy saving can be obtained by operating in beaconed mode using the superframe structure. However, this comes at the cost of significant latency and low bandwidth. The IEEE 802.15.4 beaconless mode has been studied in [16] where it is concluded that, for a single hop connection, the delay is below the $10 \mathrm{~ms}$ threshold, which seems adequate to support a ZigBee solution for the target application.

As presented, the cost of the wireless modules must be 
low in order to support a cost-effective alternative to existing CAN communications. Because the current availability of ZigBee COTS (Components Of The Shelf) supporting the beaconed mode is very limited, its use in real deployments is still not very attractive. Although there are academic endeavors (e.g. open-ZB [21]) targeting the development of opensource solutions to support the beaconed mode, their complexity is still not suitable for commercial solutions. Additionally, because these endeavors are often based on expensive hardware (e.g CrossBow MICAz or TelosB motes) and specific software (e.g. TinyOS operating system), their overall cost (the combination of development expenses and hardware costs) makes them inadequate for commercial applications, especially the those requiring cost-effectiveness such as the one addressed here.

To the best of our knowledge, there are no theoretical or experimental studies assessing the timeliness of multihop broadcast communications with ZigBee.

\section{Beaconless ZigBee}

The B-Live system operates under a producerconsumer communication model. The information is broadcasted by the producer and is received by the remaining devices, only being consumed by the appropriate ones (consumers). In this sense, the delay experienced in the network results from the propagation of the broadcast, i.e. the time it takes the broadcasted information to travel from a given producer to the corresponding consumer. Moreover, the worst case delay is defined as the amount of time that elapses between the instant when the broadcast is transmitted and the instant of its reception in the latest consumer. The worst case delay in CAN is well determined and can be far bellow the limits imposed by the application [24]

In a ZigBee network perspective, a broadcast message is successfully propagated if all devices in the network receive it (whether they are routers or end-devices). The propagation of the broadcast is influenced by several factors such as the number of hops in the path or the processing delays in the devices. Therefore, to estimate the worst case latency, all delays introduced in the communication path must be accounted.

\subsection{Broadcast Introduction}

In ZigBee, a broadcast can be initiated by any device belonging to the network. A router (or the coordinator) is able to broadcast data by instrucing its Network Layer (NWK) to request a packet transmission to the Medium Access Control (MAC) sub-layer. This procedure includes setting the destination network address to the broadcast address (0xFFFF). If the sending device is an end device, the MAC destination address is set to the 16-bit network address of its parent. This procedure establishes that, at MAC level, the communication between end device and its parent is unicast based.

In this scenario, because the medium access is based on a CSMA/CA scheme, the end-device must wait a ran-

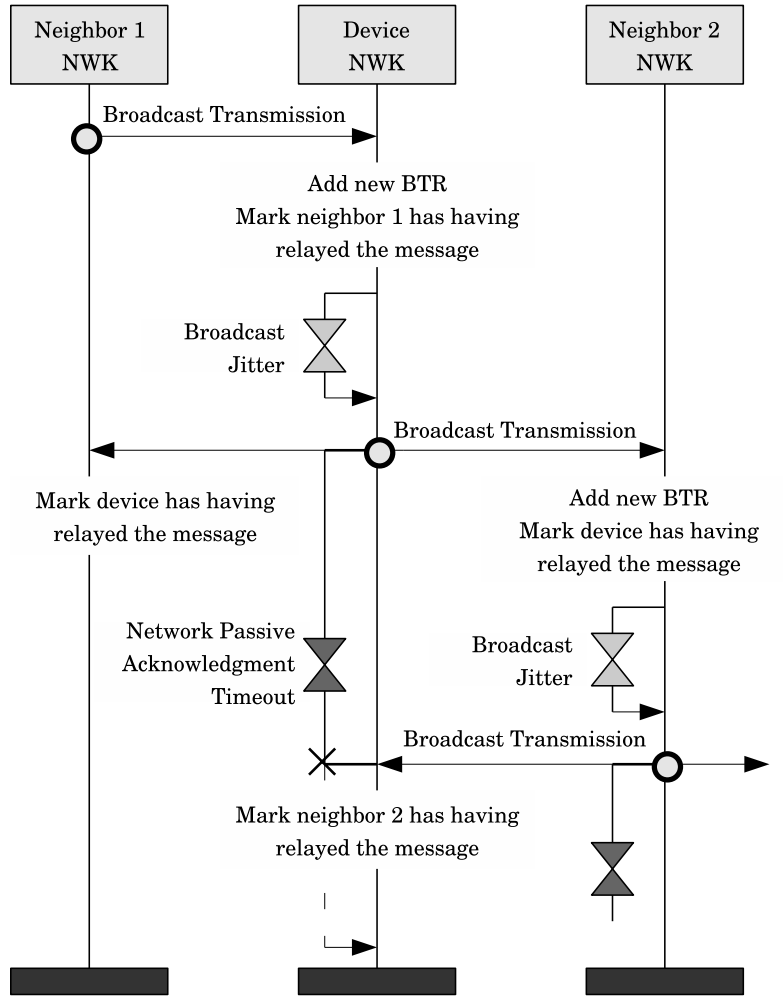

Figure 1. Acknowledgement Mechanism

dom amount of time (backoff delay) before initiating the unicast transmission. Afterwards, the MAC layer sets up a timer that expires after a given period of time (MAC Acknowledgement Wait Duration). If this period elapses before a MAC Acknowledgement is received, the unicast packet is retransmitted (up to the maximum of macMaxFrameRetries retransmissions). If a MAC Acknowledgement frame is received before the period expiration, the end device MAC assumes the packet as being correctly transmitted. When a router (or coordinator) receives a NWK broadcast from a child end device (unicast at MAC level), it performs its acknowledgement and sends the broadcast to the network.

In ZigBee networks there is no MAC acknowledgement mechanism for broadcast transmissions and a passive acknowledgement scheme is used instead. This scheme operates by keeping track if the neighboring devices have successfully relayed the broadcast transmission, as exemplified in Figure 1. The following discussion assumes a formed ZigBee network.

Routers maintain Broadcast Transaction Records (BTRs) of the broadcast transactions occurring within their radio range, which are stored in a Broadcast Transaction Table (BTT). When a device receives a broadcast packet (from a neighboring device) whose destination address does not match its own device type, the packet is discarded. Otherwise, the device compares the sequence number and the source address of the packet with the records in its BTT. If the device already has a BTR for this broadcast packet, it updates it to mark the neighboring 
device as having relayed the broadcast frame and drops the packet. If no record is found, it creates a new BTR and marks the neighboring device as having relayed the broadcast. Following, the network layer (NWK) indicates to the higher layer that a new broadcast frame has been received. ZigBee packets have a radius field that is decreased in each transmission hop. When this field reaches the value 0 , the packet is not retransmitted again.

As documented in Figure 1, before retransmitting a broadcast, a router must wait for a random amount of time named broadcast jitter. This delay reduces the probability of collisions among rebroadcasts. Just before the broadcast retransmission, a timer is set up to expire after the Network Passive Acknowledgement Timeout. As such, all neighbors should retransmit the broadcast within this period. If a neighbor fails to transmit the rebroadcast, a broadcast packet is transmitted again. This process is repeated up to nwkMaxBroadcastRetries times. As explained, if the neighbors retransmit the broadcast packet within the specified timeout, they are marked has having successfully relayed the broadcast.

\subsection{Analytical Study}

In this subsection an analytical study of the end-to-end latency of ZigBee multi-hop broadcast communications is proposed.

\section{End-to-end Latency}

The end-to-end latency is assumed to be the time elapsed between the instant when the last bit of a broadcast is transmitted at a producer node and the instant of its decoding in the last receiving consumer. This delay is dependent of the number of hops that are crossed in the communication path and, as it will be discussed further ahead, of the type of device initiating the broadcast (enddevice or router).

In Fig. 2 we show the two possible propagation scenarios. When a broadcast is produced by an end-device ( $E_{S}$ ), it is rebroadcasted by $R_{n_{r p}}$ routers until reaching its later consumer $\left(X_{D}\right.$, end-device or router $) . n_{r p}$ represents the number of routers between the producer and the consumer.

Several delays must be considered in the communication path: end-device to router link $\left(t_{E R}\right), i^{\text {th }}$ router to router link $\left(t_{R R_{i}}\right)$ and the last hop of the communication path between router $R_{i}$ and the generic device $X_{D}$ $\left(t_{R L H}\right)$. When a broadcast is produced by a router $\left(R_{S}\right)$ the scenario is similar, except for the absence of the $t_{E R}$ delay. The end-to-end delay of a broadcast initiated by an end-device is given by:

$$
t_{E 2 E_{E}}=\left\{\begin{array}{lll}
t_{R L H}^{*} & \text { if } & n_{r p}=0 \\
t_{R L H}+t_{E R} & \text { if } & n_{r p}=1 \\
t_{R L H}+t_{E R}+\sum_{i=1}^{n_{r p}-1} t_{R R_{i}} & \text { if } & n_{r p} \geq 2
\end{array}\right.
$$

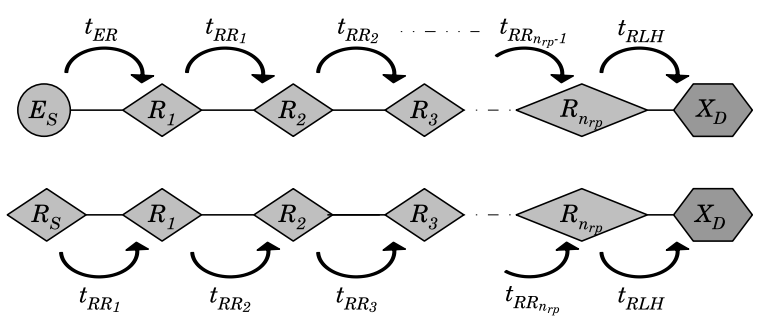

Figure 2. Broadcast paths

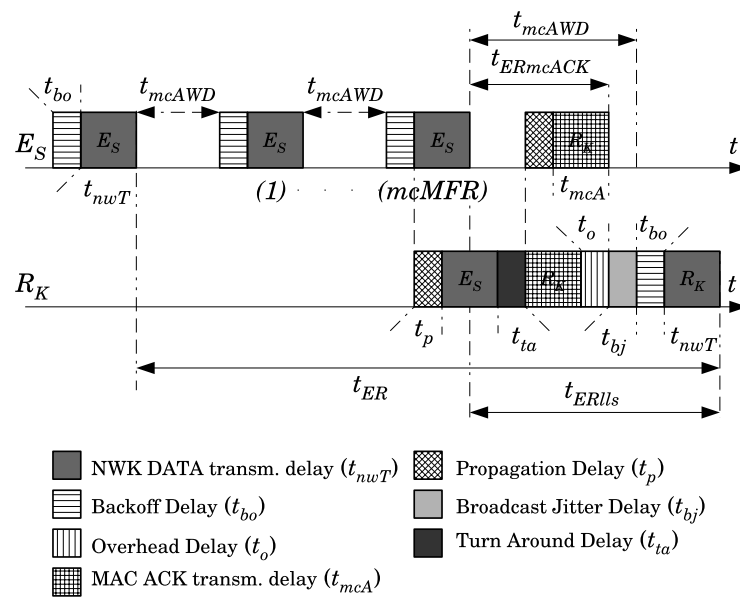

Figure 3. Broadcast by an end device

The use of a different $t_{R L H}\left(t_{R L H}^{*}\right)$ when the number of routers in the path is zero is justified by the fact that, in this scenario, the "last link" of the communication path occurs between an end-device and its parent router, not between a router and a generic device, as assumed.

The end-to-end delay for a broadcast produced by a router can be expressed as:

$$
t_{E 2 E_{R}}=\left\{\begin{array}{lll}
t_{R L H} & \text { if } & n_{r p}=0 ; \\
t_{R L H}+\sum_{i=1}^{n_{r p}} t_{R R_{i}} & \text { if } & n_{r p} \geq 1 ;
\end{array}\right.
$$

In the following text we will derive the expressions for the terms $t_{E R}, t_{R R}$ and $t_{R L H}$.

\section{Latency in a end-device to router link $\left(t_{E R}\right)$}

Fig. 3 shows the timeline of a broadcast sent by an end device $\left(E_{S}\right)$ to its parent (router $R_{K}$ ). As it is shown, the link latency $\left(t_{E R}\right)$ is assumed to be the time elapsing between the end of the broadcast transmission from the end-device and its parent. As depicted, before initiating a broadcast transmission $\left(t_{n w T}\right)$, the end device waits for a random period of time named backoff delay $\left(t_{b o}\right)$. After this time has elapsed, a Clear Channel Assessment (not represented) is performed to detect the channel state (idle/busy) in the first 8 symbol periods following the end of the backoff delay. For simplicity, we assume it as being part of the backoff delay.

The parent device must acknowledge the reception of the broadcast packet received from its child end-device. 
If the end-device receives the acknowledgement within a $t_{m c A W D}=$ macAckWaitDuration time window, the packet is not retransmitted. Otherwise, the packet is retransmitted up to $m c M F R=$ macMaxFrameRetries retries (macMaxFrameRetries $\in[0, \ldots, 7]$ ). We have represented the air propagation delay as $t_{p}$ and the duration of the acknowledge packet as $t_{m c A}$. This packet is sent immediately after the turnaround delay $\left(t_{t a}\right)$ without performing the backoff procedure.

Following the acknowledge transmission, the parent device is ready to rebroadcast the received packet. As Fig. 3 shows, we assume that the rebroadcast is delayed by an overhead latency $\left(t_{o}\right)$ caused by processing at network level. We do not represent the Inter-Frame Space (IFS) time because it is assumed to be smaller than $t_{o}$. Afterwards, the parent device delays the beginning of the rebroadcast procedure by a period of time known as broadcast jitter $\left(t_{b j}\right)$. Only after occurs the random backoff delay $\left(t_{b o}\right)$ that precedes the broadcast transmission $\left(t_{n w T}\right)$. The broadcast communication delay in a end-device to parent link is denoted by:

$$
\begin{aligned}
t_{E R} & =n_{m f r} \cdot\left(t_{m c A W D}+t_{b o}+t_{n w T}\right)+t_{E R l l s}, \\
& \forall n_{m f r} \in[0, \ldots, m c M F R]
\end{aligned}
$$

where $n_{m f r}$ represents the number of (MAC) frame retries and $t_{E R l l s}$ is the latency measured between the instant where the broadcast transmission is completed at the end device and the instant where the rebroadcast is concluded at the parent device, assuming a lossless scenario. In other words, a scenario where no broadcast retransmissions occur. This delay is given by:

$$
t_{E R l l s}=t_{p}+t_{t a}+t_{m c A}+t_{o}+t_{b j}+t_{b o}+t_{n w T}
$$

\section{Latency in a router to router link $\left(t_{R R}\right)$}

The timeline of a broadcast initiated by a router is represented in Fig. 4. It is shown that, if the broadcast is not passively acknowledged by all neighboring routers within $t_{n w P A T}=n w k P a s s i v e A c k T i m e o u t$ seconds (nwkPassiveAckTimeout $\in[0, \ldots, 10])$, the broadcast is retransmitted up to a maximum of $n w M B R=n w k M a x B r o a d c a s-$ tRetries times (nwkMaxBroadcastRetries $\in[0, \ldots, 5]$ ). As before, the packet is affected by several delays: propagation $\left(t_{p}\right)$, network layer overhead $\left(t_{o}\right)$, broadcast jitter $\left(t_{b j}\right)$, backoff $\left(t_{b o}\right)$ and transmission $\left(t_{n w T}\right)$. The broadcast communication delay between two routers is given by the following expression:

$$
\begin{gathered}
t_{R R}=n_{n b r} \cdot\left(t_{n w P A T}+t_{b o}+t_{n w T}\right)+t_{R R l l s}, \\
\forall n_{n b r} \in[0, \ldots, n w M B R]
\end{gathered}
$$

where $n_{n b r}$ represents the number of (NWK) broadcast retries and $t_{R R l l s}$ the latency between two routers in a lossless scenario, as represented by:

$$
t_{R R l l s}=t_{p}+t_{o}+t_{b j}+t_{b o}+t_{n w T}
$$

Latency in the last link of the path $\left(t_{R L H}\right.$ or $\left.t_{R L H}^{*}\right)$
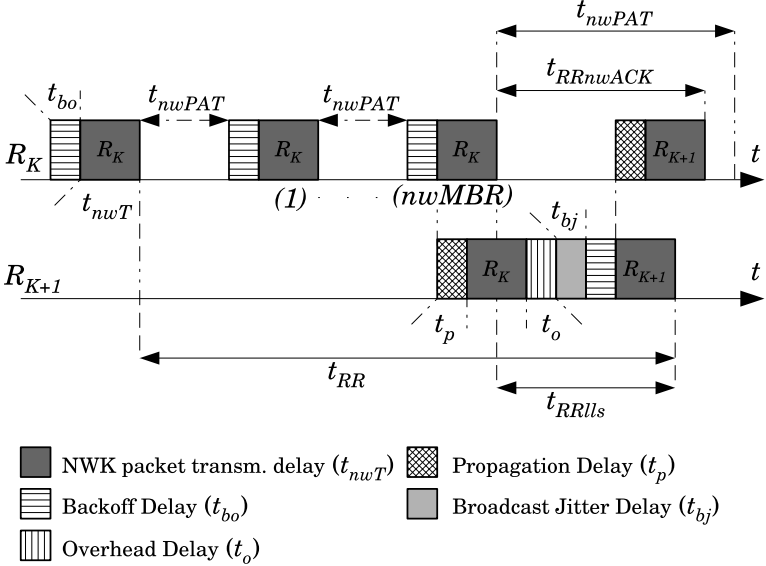

Figure 4. Broadcast by a router

Recalling Fig. 4, the latency experienced in the last hop of the propagation path $\left(t_{R L H}\right)$, connecting a router to a receiving device (end-device or router), is the time elapsing from the end of the broadcast transmission at the router and the instant where its is received in the consumer device, as expressed by:

$$
\begin{gathered}
t_{R L H}=n_{n b r} \cdot\left(t_{n w P A T}+t_{b o}+t_{n w T}\right)+t_{R L H l l s}, \\
\forall n_{n b r} \in[0, \ldots, n w M B R]
\end{gathered}
$$

where $n_{n b r}$ expresses the number of broadcast retries and $t_{R L H l l s}$ the latency in the last communication hop considering a lossless scenario where no retransmissions occur. This latency is given by:

$$
t_{R L H l l s}=t_{p}+t_{o}
$$

When a broadcast is produced by an end-device and there is only one hop in the communication path, a special scenario arises. This scenario resembles the broadcast propagation between an end-device and its parent except that the delay is measured up to the instant where the broadcast is received instead of the instant where it is rebroadcasted by the parent. Recalling Fig. 3, this latency $\left(t_{R L H}^{*}\right)$ is given by:

$$
\begin{aligned}
t_{R L H}^{*} & =n_{m f r} \cdot\left(t_{m c A W D}+t_{b o}+t_{n w T}\right)+t_{R L H l l s}, \\
& \forall n_{m f r} \in[0, \ldots, m c M F R]
\end{aligned}
$$

\section{Assessment}

In order to validate the proposed theory, an assessment of the broadcast latency is performed by measuring the end-to-end delay on a ZigBee network with a controlled topology and external interference.

\subsection{Test-Bed}

A ZigBee network was deployed in the ground floor of Micro I/O [1]. This network is composed of four 
routers: a coordinator that is also the data source $\left(R_{S}\right)$, a sink router $\left(R_{D}\right)$, and two path routers $\left(R_{1}\right.$ and $\left.R_{2}\right)$ that 'link' the source to the sink. These routers are placed in a straight line $\left(R_{S}, R_{1}, R_{2}, R_{D}\right)$ so that they only communicate with their direct neighbors ( $R_{S}$ with $R_{1}, R_{1}$ with $R_{2}$ and $R_{S}$, etc.).

The used ZigBee (MioBee) devices are based on a simple hardware architecture: a board housing a Microchip [19] PIC18F2580 microcontroller, a MaxStream [18] XBee module and oscillator, pull-up resistors, etc.

The firmware running in the microcontroller of the source device (coordinator) is different from the remaining devices since it periodically produces an API Data frame and sends it to the attached XBee module through an EIA-232 connection. This message is then processed and a ZigBee broadcast packet is sent over-the-air. When this packet is received by a neighbor XBee module, an API Data frame is forwarded by the module to the attached microcontroller through the EIA-232 connection. Because the received packet is a broadcast, the XBee module proceeds with its retransmission to (passively) acknowledge the "original" broadcast. The firmware running in the microcontroller of the routers $R_{1}$ and $R_{2}$ was designed to configure the module's parameters at boot time and ignore any received API messages at runtime, as the rebroadcast mechanism works entirely on the XBee modules.

In order to assess the timeliness of multi-hop ZigBee broadcast communications we induce the occurrence of collisions by placing a $802.11 \mathrm{~b}$ (Wi-Fi) network [13] in range, configured on a channel overlapping the used ZigBee channel. Three Wi-Fi data sources (2 PCs with 3Com PCI 3CRDW696 cards plus one laptop with a 3Com PCMCIA 3CRSHPW796 card) were placed within the range of the ZigBee network and connected in infrastructure mode to a Cisco Aironet 1200 Access Point. The load on the Wi-Fi network was generated using the Distributed Internet Traffic Generator (D-ITG) [4].

The measurement of the broadcast end-to-end latency was performed using a Delay Measurement System (DMS) [7]. The DMS operates by registering the transmitting and receiving instants, at the producer and the consumer, respectively, and computing the corresponding latencies. A delay histogram and measures of minimum, average and maximum delay, number of transmitted and received data bytes are provided by the measurement system.

\subsection{Results}

This subsection presents both theoretical and experimental results as well as their discussion.

\section{Analytical}

Fig. 5 shows the behavior of the broadcast delay in the proposed test-bed scenario as a function of the total number of broadcast retransmissions in the communication path. This result was obtained using Eq. 2 with the following assumptions:

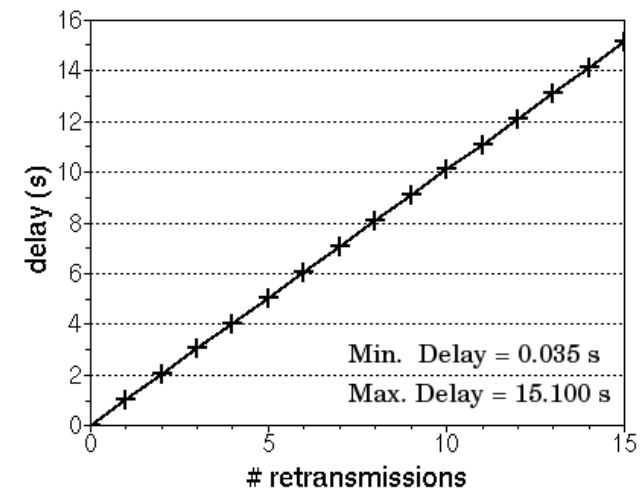

Figure 5. 3-hop end-to-end broadcast delay (initiated by a router)

a) Typical backoff exponent (3) for a null number of retransmissions. This value is incremented by 1 (up to 5) for each retransmission.

b) Retransmissions occur in a hop by hop basis, i.e. only after the first hop have reached 5 retransmissions (maximum value), there will be retransmissions in the second hop, and so on.

c) The backoff delay $\left(t_{b o}\right)$ is determined considering the average number of possible slots. For example, if the backoff exponent is 4 , the number of backoff slots considered is $\left(2^{4}-1\right) / 2=7.5$ slots.

d) Fixed parameters: two routers in the broadcast path $\left(n_{r p}=2\right)$, average broadcast jitter $\left(t_{b j}=32 \mathrm{~ms}\right)$, null propagation and overhead delays $\left(t_{p}=0 \mathrm{~s}\right.$, $\left.t_{o}=0 \mathrm{~s}\right)$, minimum network Passive Acknowledgment Timeout $\left(t_{n w P A T}=1 \mathrm{~s}\right)$ and broadcast messages with a physical length of 48 octets and a transmission delay $\left(t_{n w T}\right)$ of $1.536 \mathrm{~ms}$.

As expected, the end-to-end delay increases with the number of retransmissions. The minimum delay (35 ms) is obtained in the absence of broadcast retransmissions while the maximum delay (15.1 s) arises when the maximum number of retransmissions has occurred for a valid end-to-end communication. The (large) variation in the end-to-end delay results from the influence of the network Passive Acknowledgement Timeout $\left(t_{n w P A T}\right)$ and of the number of retransmissions. Because this timeout is given in seconds, when a broadcast transmission fails to be acknowledged by one (or more) of the neighboring routers, the sender must wait a large amount of time before initiating the rebroadcast. This timeout is defined by the ZigBee Alliance to cope with broadcast communications in large networks. Clearly, it was not specified envisaging common home automation applications.

\section{Experimental}

To confirm the theoretical analysis, an experimental evaluation was conducted using the previously described 
Table 1. Trial Parameters

\begin{tabular}{|l|l|}
\hline \hline \multicolumn{2}{|l|}{ ZigBee } \\
\hline Channel & 18 \\
\hline Broadcast period & $4 s$ \\
\hline Broadcast radius & 10 \\
\hline Payload length & (constant) 1 byte \\
\hline Data Rate & $250 \mathrm{Kbps}$ \\
\hline \multicolumn{2}{|l|}{ Wi-Fi } \\
\hline Channel & 6 \\
\hline & $0,2,4,6,8,10,12,14,16$, \\
Offered Loads $(\%)$ & $18,20,22,24,26,28,30,35$, \\
& $40,45,50,55,60,70,80,90$, \\
& 100 \\
\hline Data Rate & $11 \mathrm{Mbps}$ \\
\hline Tx. Power & $5 \mathrm{~mW}$ \\
\hline Payload length & (constant) 1 or 1472 bytes \\
\hline \hline
\end{tabular}

test-bed with the parameters shown in Table 1.

Several trials were conducted with different offered loads and packet lengths. These loads were calculated considering the physical length of 802.11 PHY frames and including the Inter Frame Space as transmission time. In each trial, the source device $\left(R_{S}\right)$ broadcasts $200 \mathrm{ZigBee}$ data packets, which are then rebroadcasted by neighbor devices $\left(R_{1}\right.$ and $\left.R_{2}\right)$ until reaching the target device $\left(R_{D}\right)$. For each successfully transmitted packet (end-to-end), the DMS updates a the related statistics.

Fig. 6 shows the percentage of lost packets with the offered Wi-Fi load (for both 1 and 1472 bytes Wi-Fi payloads). As expected, the Packet Error Rate (PER) increases with the offered load. This occurs because the number of collisions becomes larger as the traffic load increases.

Another visible aspect is the different behavior resulting from using maximum or minimum payload packets in the Wi-Fi traffic. For a given offered load, the number of short packets is higher than of large packets. Therefore, Wi-Fi stations transmitting short packets are able to gain access to the medium more often. Since the 802.15.4/ZigBee CSMA/CA mechanism will also detect the medium occupied more often, the PER is higher in the presence of Wi-Fi short packet communications. For offered loads above $60 \%$, there are no broadcast communications for either Wi-Fi packet lengths.

Fig. 7 and Fig. 8 show the minimum and maximum delays for different Wi-Fi loads. Trials with no successful transmissions are represented by an infinite latency. As it can be seen, in the absence of interference, both minimum and maximum delays are small (35.77 $\mathrm{ms}$ and $96.83 \mathrm{~ms}$, respectively) and within the bounds defined for the application (delay $\leq 500 \mathrm{~ms}$ and jitter $\leq 100 \mathrm{~ms}$ ).

When the offered load increases up to $55 \%$, we observe that the minimum delays range from $32.63 \mathrm{~ms}$ to $63.3 \mathrm{~ms}$ and the maximum delays from $962.35 \mathrm{~ms}$ to $2183.7 \mathrm{~ms}$. From Fig. 7, it is clear that the minimum delay oscillates around the $35 \mathrm{~ms}$ value, which matches the minimum delay shown in Fig. 5. Regarding Fig. 8, the maximum de-

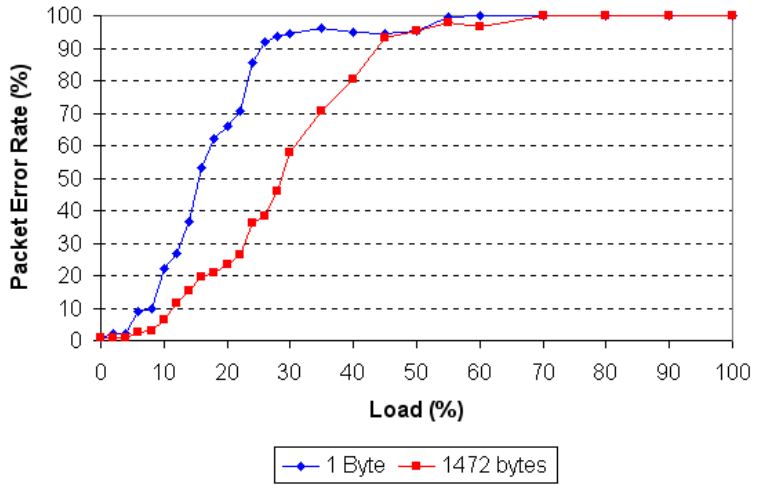

Figure 6. Packet Error Rate vs. Offered Load

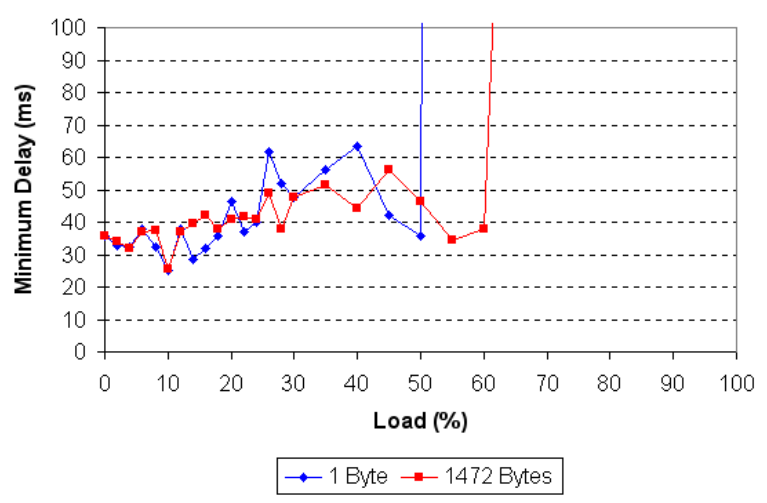

Figure 7. Minimum Delay $v s$. Offered Load

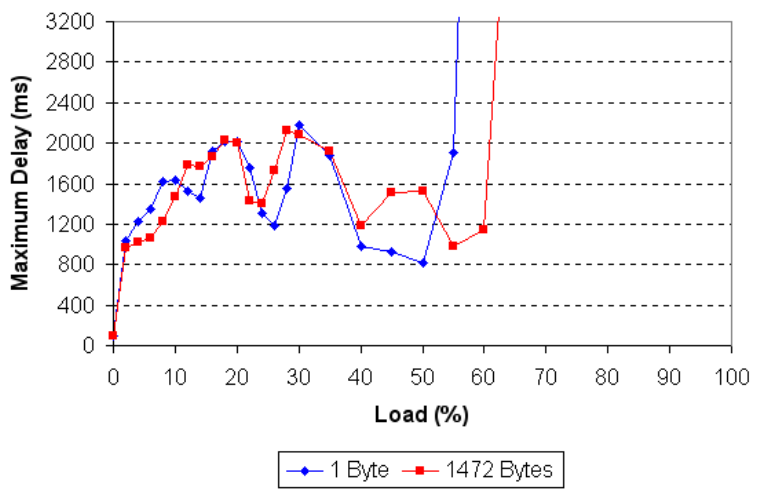

Figure 8. Maximum Delay vs. Offered Load

lay oscillates within 1 and 2 seconds, which seems aligned with the delays documented in Fig. 5 for 1 and 2 broadcast retransmissions, respectively.

As seen in Fig. 8, we were not able to obtain large delays ( $\geq 3 \mathrm{~s}$ ) to compare with the theoretical analysis. This may result from the short length of the trials and from the restrictive set of conditions leading to a small number of retransmissions (without packet loss).

\section{Conclusion}

Little attention has been devoted to the support of realtime communications using ZigBee. In this paper, a theoretical analysis and an experimental validation of the endto-end delay in ZigBee multi-hop broadcast communica- 
tions is performed.

The experimental evaluation shows that, in interference-free environments, the minimum and maximum delays for a three-hop communication path are $35.77 \mathrm{~ms}$ and $96.83 \mathrm{~ms}$, respectively. The conclusion is that the timeliness of ZigBee broadcast communications seems adequate to building automation systems of reduced size when the network operates without retransmissions (in free RF channels).

Future work includes inducing controlled retransmissions and perform a comprehensive verification of the derived analytical expressions. The impact of high event rates (observed in alarm scenarios) in the timeliness of ZigBee broadcast communications should also be studied as well as issues regarding reliability and security. Furthermore, in the course of extending the application domain, energy consumption should be addressed.

\section{Acknowledgement}

The authors would like to thank José Oliveira for conducting most of the experimental trials and to Micro I/O for making the facilities available to perform the trials.

Paulo Bartolomeu is supported by Fundação para a Ciência e a Tecnologia under grant POSI 2004 Medida 6.1, Ref. SFRH/BDE/15602/2006 and by Micro I/O.

This work was partially supported by NITEC/114/20/06 (71/00101), TIGELA, Programa PRIME, funded by ADI (Agência de Inovação).

\section{References}

[1] Micro I/O - Sistemas de Electrónica, Lda. http://www.microio.pt, March 2008.

[2] Z. Alliance. Document 053474r13. ZigBee Specification, pages i-508, December 2006.

[3] Z. Alliance. http://www.zigbee.org, March 2008.

[4] S. Avallone and A. Pescapè. Distributed Internet Traffic Generator (D-ITG) Version 2.6.1c . http://www.grid.unina.it/software/ITG/, March 2008.

[5] P. Bartolomeu, J. Fonseca, V. Santos, A. Mota, V. Silva, and M. Sizenando. Automating Home Appliances for Elderly and Impaired People: The B-Live Approach. In 2nd International Conference on Software Development for Enhancing Accessibility and Fighting Infoexclusion, pages 65-72, 2007.

[6] P. Bartolomeu, J. Fonseca, and F. Vasques. Challenges in Health Smart Homes. In Workshop on Ambient Technologies for Diagnosing and Monitoring Chronic Patients, part of the 2nd International Conference on Pervasive Computing Technologies for Healthcare, 2008.

[7] P. Bartolomeu, V. Silva, and J. Fonseca. Delay measurement system for real-time serial data streams. Emerging Technologies \& Factory Automation, 2007. ETFA. IEEE Conference on, pages 516-523, September 2007.

[8] R. Bosh. Can specification version 2.0. BOSCH GmbH, September 1991.

[9] J. Coughlin. Technology needs of aging boomers. Issues in Science and Technology, 16(1):53-60, 1999.

[10] G. Ding, Z. Sahinoglu, B. Bhargava, P. Orlik, and J. Zhang. Reliable broadcast in ZigBee networks. Sensor and Ad Hoc Communications and Networks, 2005.
IEEE SECON 2005. 2005 Second Annual IEEE Communications Society Conference on, pages 510-520, 2005.

[11] G. Ding, Z. Sahinoglu, P. Orlik, J. Zhang, and B. Bhargava. Tree-based data broadcast in IEEE 802.15.4 and ZigBee networks. Mobile Computing, IEEE Transactions on, 5(11):1561-1574, 2006.

[12] J. A. Gutierrez, E. H. Callaway, and R. Barrett. IEEE 802.15.4 Low-Rate Wireless Personal Area Networks: Enabling Wireless Sensor Networks. IEEE Standards Office, New York, NY, USA, 2003.

[13] IEEE. Wireless Lan Medium Access Control (MAC) And Physical Layer (PHY) Specifications. IEEE Std 802.111997, pages i-445, 1997.

[14] IEEE. Wireless Medium Access Control (MAC) and Physical Layer (PHY) Specifications for Low-Rate Wireless Personal Area Networks (WPANs). IEEE Std 802.15.42006, pages i-305, 2006.

[15] A. Koubâa, M. Alves, and E. Tovar. A comprehensive simulation study of slotted CSMA/CA for IEEE 802.15.4 wireless sensor networks. Factory Communication Systems, 2006 IEEE International Workshop on, pages 183192, 2006.

[16] B. Latr, P. D. Mil, I. Moerman, B. Dhoedt, P. Demeester, and N. V. Dierdonck. Throughput and delay analysis of unslotted IEEE 802.15.4. Journal of Networks, 1(1):2028, 2006.

[17] G. Lu, B. Krishnamachari, and C. Raghavendra. Performance evaluation of the IEEE 802.15.4 MAC for low-rate low-power wireless networks. Performance, Computing, and Communications, 2004 IEEE International Conference on, pages 701-706, 2004.

[18] MaxStream. http://www.maxstream.net/, April 2008.

[19] Microchip. http://www.microchip.com, April 2008.

[20] S.-Y. Ni, Y.-C. Tseng, Y.-S. Chen, J.-P. Sheu, and J.-P. Sheu. The broadcast storm problem in a mobile ad hoc network. In MobiCom '99: Proceedings of the 5th annual ACM/IEEE international conference on Mobile computing and networking, pages 151-162, USA, 1999. ACM.

[21] Open-ZB. Opensource toolset for 802.15.4 and zigbee. http://www.open-zb.net, April 2008.

[22] B. Shneiderman. Response time and display rate in human performance with computers. ACM Comput. Surv., 16(3):265-285, 1984.

[23] I. Stojmenovic and J. Wu. Broadcasting and activityscheduling in ad hoc networks. In S. Basagni, M. Conti, S. Giordano, and I. Stojmenovic, editors, Ad Hoc Networking. IEEE Press, 2004.

[24] K. Tindell, A. Burns, and A. J. Wellings. Calculating Controller Area Network (CAN) message response times. Control Engineering Practice, 3(8):1163-1169, 1995.

[25] E. Vollset and P. Ezhilchelvan. A survey of reliable broadcast protocols for mobile ad-hoc networks. Technical report, University of Newcastle, 2003.

[26] J. Zheng and M. J. Lee. A comprehensive performance study of IEEE 802.15.4. In Sensor Network Operations, pages 218-237. IEEE Press, Wiley Interscience, 2006. 